REVIEW

\title{
A critical review and meta-analysis of the association between overt hyperthyroidism and mortality
}

\author{
Frans Brandt, Anders Green ${ }^{1,2}$, Laszlo Hegedüs and Thomas H Brix \\ Department of Endocrinology and Metabolism, Odense University Hospital, Klovervaenget 6, 5000 Odense C, Denmark, ${ }^{1}$ Research Unit of Clinical \\ Epidemiology, University of Southern Denmark, Odense, Denmark and ${ }^{2}$ Center for National Clinical Databases, South, Odense University Hospital, \\ Odense, Denmark \\ (Correspondence should be addressed to F Brandt; Email: fbrandt@health.sdu.dk)
}

\begin{abstract}
Background: Overt hyperthyroidism has been associated with cardiac arrhythmias, hypercoagulopathy, stroke, and pulmonary embolism, all of which may increase mortality. Some, but not all, studies show an increased mortality in patients with hyperthyroidism. This inconsistency may be due to differences in study design, characteristics of participants, or confounders. In order to test whether hyperthyroidism influences mortality, we performed a critical review and statistical meta-analysis. Methods: Based on an electronic PubMed search, using the Medical Subject Heading words such as hyperthyroidism, thyrotoxicosis, and mortality or survival, case-control and cohort studies were selected and reviewed. Using meta-analysis, an overall relative risk (RR) of mortality was calculated. Results: Eight studies fulfilled the inclusion criteria, six of which showed an increased all-cause mortality; seven studies, including 31138 patients and 400000 person years at risk, allowed calculation of mortality in a meta-analysis. Based on this, the RR of overall mortality was 1.21 (95\% confidence interval: 1.05-1.38). Analyses including studies considering setting, treatment, and control for co-morbidity did not significantly alter this finding. As the measured heterogeneity $\left(\mathrm{I}^{2}\right)$ ranges from 89.1 to $98.3 \%$, which is much higher than the $50 \%$ generally viewed on as a threshold, the statistical heterogeneity is very pronounced in the included studies.

Conclusion: In patients diagnosed with hyperthyroidism, mortality is increased by $\sim 20 \%$. Future studies need to address the cause of hyperthyroidism, impact of type of therapy, time dependency, as well as the potential influence of confounding or genetic susceptibility before the question of causality can be answered.
\end{abstract}

European Journal of Endocrinology 165 491-497

\section{Introduction}

Overt hyperthyroidism is common and affects $2-5 \%$ of the population $(1,2)$. Hyperthyroidism is due to complex interactions between genetic susceptibility and environmental triggers such as the level of iodine intake and magnitude of smoking. Biochemically, the disorder is defined by a suppressed serum level of TSH and elevated concentrations of the thyroid hormones thyroxine and/or triiodothyronine. Irrespective of its cause (Graves' disease or nodular toxic goiter), hyperthyroidism is associated with conditions such as atrial fibrillation (3), stroke (4), pulmonary embolism (5), and hypercoagulopathy (6), which, theoretically, could lead to an increased mortality. Several studies have investigated the relationship between overt hyperthyroidism and mortality. Nevertheless, conflicting data from these studies have led an ongoing debate regarding the consequences of overt hyperthyroidism with respect to mortality. This inconsistency has been explained by differences in study design, characteristics of participants, treatment effect and modalities, or the influence of confounding factors (7).

In order to evaluate whether there is an association between overt hyperthyroidism and mortality, we performed a critical review and meta-analysis using the available data.

\section{Methods}

\section{Search method}

On October 1st 2010, we performed a MEDLINE database search using the PubMed search engine with the Medical Subject Heading (MeSH) words such as hyperthyroidism or thyrotoxicosis and mortality or survival. Only abstracts written in English were taken into account, but there were no restrictions with respect to publication date, treatment modality, study design, study setting (hospital or primary health care), gender, or age. Abstracts were reviewed independently by two 
authors (F B and T H B), and differences were resolved by discussion. Inclusion criteria were case-control or cohort studies published in peer-reviewed journals, investigating whether overt hyperthyroidism is associated with mortality. Exclusion criteria were duplication of data, if patients were not diagnosed with overt hyperthyroidism (e.g. subclinical hyperthyroidism), or lack of a relevant control group. Furthermore, to avoid missing any relevant study, the reference lists of studies included in this review were screened in search of overlooked publications. Characteristics of the participants, type of intervention, study design, and confounders were obtained from the included studies.

\section{Analysis of studies}

In order to evaluate homogeneity and identify bias, all included studies were compared with focus on selection of patients, reliability of diagnosis (self-reported or biochemical testing), comparability of the control groups (age, gender, and morbidity), type of treatment, and major confounders (smoking and co-morbidity). In case of an overlap of subjects in two or more studies, the largest study was used for further analysis. By combining relevant studies for the meta-analysis, we evaluated various clinically relevant scenarios such as whether the studies were hospital or primary care based, only radioiodine or all treatments were chosen for therapy, and whether co-morbidity (often cardiovascular disease, diabetes, and cancer) was registered. This procedure was used instead of quality-rating scales, since none of them has been shown to be superior to analysis based on clinical relevant parameters (8). Unfortunately, it was not possible to reevaluate the studies in respect to disease phenotype, time point of diagnosis, and effectiveness of treatment since generally no such data could be obtained from the included studies.

\section{Statistical analysis}

Because of substantial qualitative and quantitative differences across studies, all analyses were performed using a random effect model (assumes that all studies are heterogeneous). The number of deaths and number of expected deaths were extracted from eligible studies and the relative risk (RR) of mortality, with 95\% confidence interval (CI), was calculated by using the method of DerSimonian \& Laird (9). Results were significant when the range of the $95 \%$ CI excluded the value 1 . To avoid an overestimate of mortality, patients lost to follow-up were considered as being alive. The statistical heterogeneity was assessed by the squared-I value, which describes the total variation across studies attributable to heterogeneity rather than to chance. A value above 25,50 , and $75 \%$ indicates low, moderate, and high heterogeneity respectively (10). We assessed publication bias by Egger's regression test (11).
Significant differences were defined as a $P$ value $<0.05$ using a two-tailed test. All analyses were carried out using version 11 of the STATA statistical package (StataCorp., College Station, TX, USA).

\section{Results}

\section{Selection and characteristics of the studies}

As outlined in Fig. 1, 551 studies were identified by a PubMed search using the MeSH words such as hyperthyroidism or thyrotoxicosis and mortality or survival. In total, 19 studies matched our additional

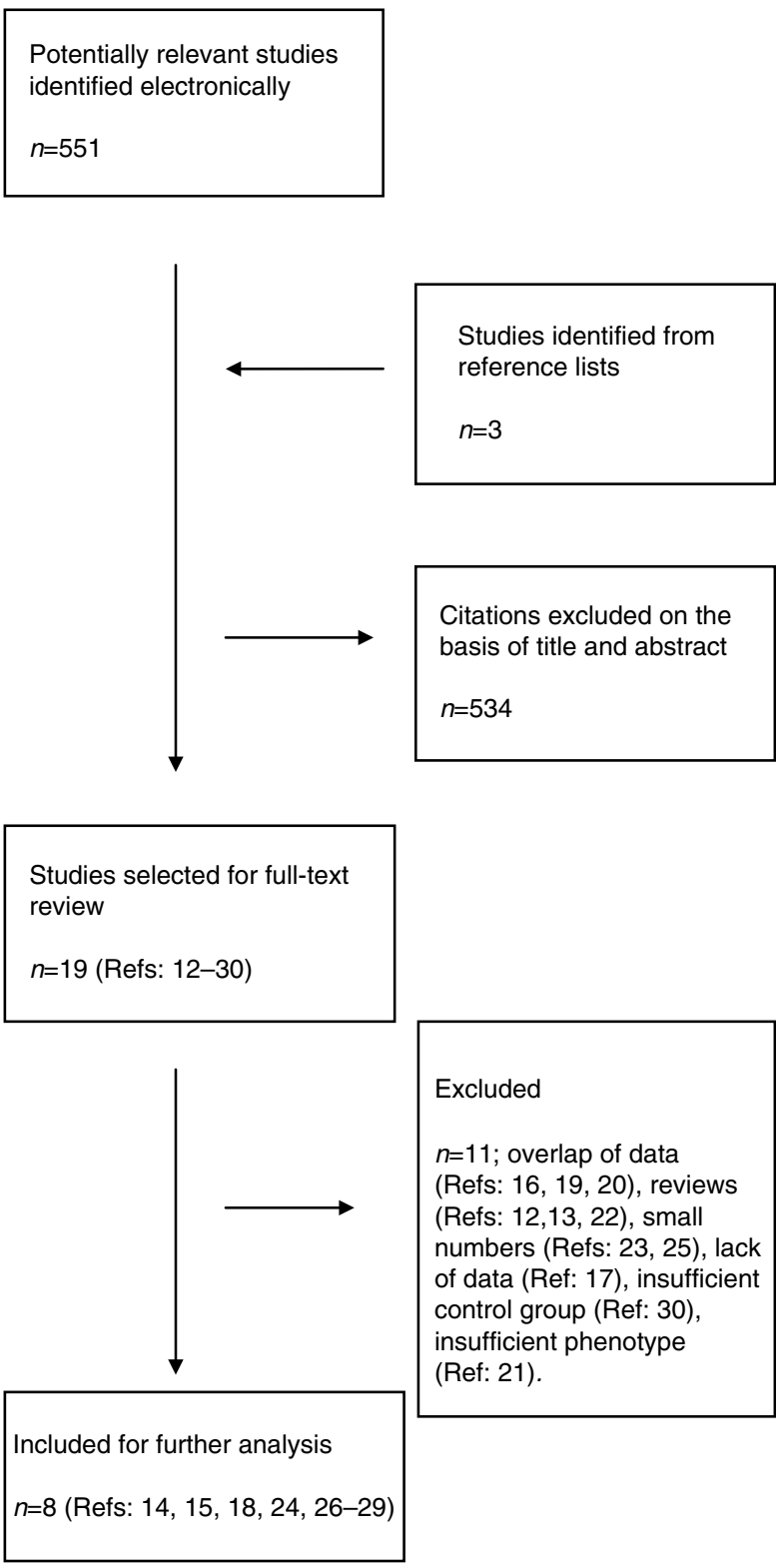

Figure 1 Flow chart outlining the process of search criteria and study selection. Refs, References. 
criteria (case-control or cohort studies) (12-30). Following review, studies were excluded because of an overlap with subjects from other studies $(16,19,20)$; because reviews were not providing original data $(12$, 13, 22); due to inclusion of very few $(n<10)$ hyperthyroid individuals to meaningfully allow calculation of the mortality risk $(23,25)$; because of lack of overall mortality data (17); based on inclusion of a control group that was also hyperthyroid (30); or, finally, because evaluation of thyroid status was solely based on serum TSH (21) (Fig. 1). Details of the remaining eight $(14,15,18,24,26-29)$ studies on which our review is based are shown in Table 1 .

Seven of the eight studies are cohort studies $(14,15$, $18,24,26,28,29)$ and one is a case-control study (27). In most studies, patients were ascertained from registers, where they have been either listed due to treatment with radioiodine $(15,18,24,29)$ or based on treatment of hyperthyroidism at a hospital unit (14) or in primary care (26). In one study, patients participated in an osteoporosis survey of middle-aged women where information on thyroid status was self-reported using questionnaires (28).

In the majority of studies, the clinical features and underlying cause or severity of hyperthyroidism were not reported in detail. Most studies were performed in Europe, either in the UK $(18,24,26,27)$ or in Scandinavia $(15,29)$. Two studies were from the USA $(14,28)$. The first study was published in $1990(14)$ and the last three in 2007 (27-29). The largest study comprised 10646 persons (15), amounting to 152406 person years at risk, while the smallest was a case-control study with 393 individuals (27). In four studies, the patients were treated with radioiodine $(15,18,24,29)$, whereas all therapy options were used in another four studies (14, 26-28). All surveys took age and sex into consideration as potential confounders, while smoking was considered in only three studies $(14,27,28)$. Selected co-morbidities, such as co-existing diabetes or cardiovascular disease, were taken into account in four studies $(14,26,27,29)$. Although all studies included an age- and gender-matched control group, the choice of control population was very inhomogeneous. Most studies calculated expected death rates based on national death registers $(14,15$, $18,26)$ or have chosen controls from a population register (29). On the other hand, controls were also included from hospital employees (27), a group of women over 65 years of age included in an osteoporosis survey (28), or patients treated in the same year as the cases, for a non-thyroid disease at a hospital (24).

\section{Mortality}

Six of the eight studies reported a higher mortality in the hyperthyroid patients compared with the corresponding control group $(14,15,18,27-29)$ while no or a non-significant difference was reported in two other

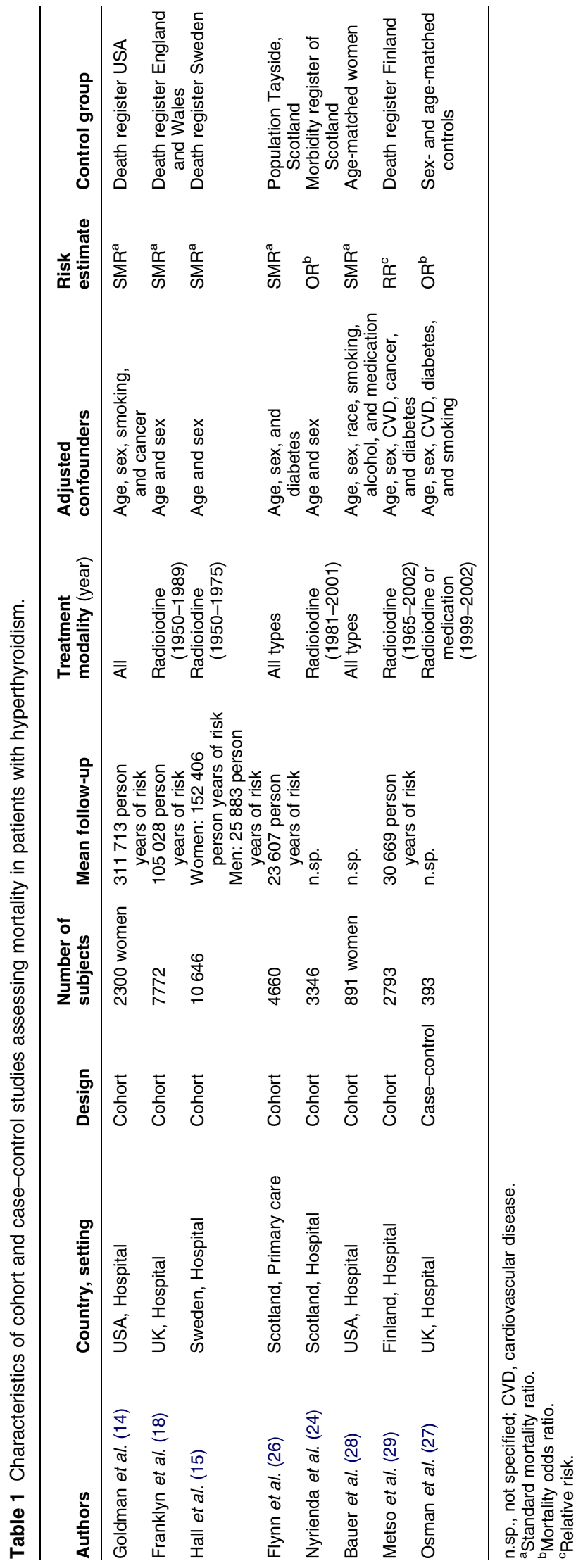

www.eje-online.org 
studies $(24,26)$. Interestingly, the only two studies, which failed to show a significant increase in mortality risk, were in contrast to other studies, either performed in primary care (26) or included controls from a hospital morbidity register after they had been admitted with a non-thyroid disease (24).

Seven of the eight studies could be pooled as only one study (27) did not provide the exact count of deaths but only risk estimates. Together, these seven studies comprised 31138 patients corresponding to $\sim 400000$ person years at risk. Irrespective of whether all studies $(1.21,95 \%$ CI $1.05-1.38)$, only studies controlling for co-morbidity (1.21, 95\% CI 1.01-1.44), or only studies performed in a hospital setting (1.24, 95\% CI 1.07-1.44), were pooled, there was a significantly higher mortality in the hyperthyroid group. An increased, but statistically non-significant, mortality risk remained when pooling all studies treating with radioiodine (Table 2 and Fig. 2).

Six studies $(14,15,18,26,27,29)$ reported cardiovascular mortality, four of which found a significantly raised mortality $(14,18,27,29)$. When pooling these six studies, there was a higher mortality in subjects with hyperthyroidism compared with the control population (RR 1.19; 95\% CI 1.00-1.29).

As the squared-I ranges from 89.1 to $98.3 \%$, which is much higher than the $50 \%$ generally viewed on as a threshold, the statistical heterogeneity is very pronounced in the included studies (Fig. 2). On the other hand, no evidence of publication bias was detected (Egger's test, $P=0.409$ ).

\section{Discussion}

Six of the eight eligible studies showed significantly increased overall mortality. A trend toward higher mortality in patients with hyperthyroidism compared with controls was also found in the remaining two studies. Our meta-analysis based on seven studies suggests a $20 \%$ increase in mortality in patients with hyperthyroidism. Thus, there is undoubtedly a statistically significant association between hyperthyroidism and increased mortality. The key question, however, is whether this increase is due to the hyperthyroidism per se or other factors such as smoking, co-morbidity, or the presence of confounders.

Clearly, the optimal study for investigating whether the observed association between hyperthyroidism, the consequence of different treatment modalities, and mortality is causal would be a randomized - as for treatment - controlled long-term follow-up study. However, such studies are not available and are rarely feasible, primarily for ethical reasons. It follows that other means, using less advantageous designs, to build up evidence for or against cause must be employed. In practice, it is widely accepted to differentiate between causal and non-causal relations by evaluating the following aspects of an association: strength, consistency, specificity, temporality, dose-response relation, and biological plausibility (31) - the so-called Bradford Hill criteria.

Our finding of a $20 \%$ raised mortality indicates a rather strong association. Despite differences in methodology, most studies show an increased mortality, reflecting a high degree of consistency. Moreover, as death is the final outcome, all studies show the right temporality. In contrast, as hyperthyroidism has been associated with a number of potentially lethal conditions such as structural changes in the heart (32), thromboembolic episodes $(4,5)$, and cardiac arrhythmias (3), the relation between hyperthyroidism and mortality is biologically plausible but clearly nonspecific. As described in case reports, severe hyperthyroidism, as seen in thyrotoxic crisis, can be lethal (33). On the other hand, a recent meta-analysis failed to show a significant association between subclinical hyperthyroidism and all-cause mortality (34). Unfortunately, in clinically overt hyperthyroidism, it has not been evaluated whether there is a relationship between the severity of the hyperthyroidism and mortality (14, $15,18,24,26-29)$. Therefore, the question of a doseresponse relationship - while plausible - remains unanswered. Although the precise mechanism(s) by which hyperthyroidism leads to an increased mortality remain to be defined, hyperthyroidism has been

Table 2 Number of deaths/expected deaths and calculated relative risk of mortality.

\begin{tabular}{|c|c|c|c|c|c|}
\hline Authors & $\begin{array}{c}\text { Observed } \\
\text { number of } \\
\text { deaths }\end{array}$ & $\begin{array}{c}\text { Expected } \\
\text { number of } \\
\text { deaths }\end{array}$ & $\begin{array}{c}\text { Observed number } \\
\text { of cardiovascular } \\
\text { deaths }\end{array}$ & $\begin{array}{c}\text { Expected number } \\
\text { of cardiovascular } \\
\text { deaths }\end{array}$ & RR $(95 \% \mathrm{Cl})$ \\
\hline Goldman et al. (14) & 790 & 564 & 359 & 256 & $1.40(1.28,1.53)$ \\
\hline Franklyn et al. (18) & 3611 & 3186 & 1955 & 1577 & $1.13(1.09,1.17)$ \\
\hline Hall et al. (15) & 5400 & 3673 & 3274 & 1984 & $1.47(1.42,1.52)$ \\
\hline Flynn et al. (26) & 565 & 539 & 386 & 368 & $1.05(0.94,1.17)$ \\
\hline Nyrienda et al. (24) & 568 & 548 & n.sp. & n.sp. & $1.04(0.94,1.15)$ \\
\hline Bauer et al. (28) & n.sp. & n.sp & n.sp & n.sp. & Not calculated \\
\hline Metso et al. (29) & 1390 & 1299 & 794 & 697 & $1.07(1.01,1.13)$ \\
\hline Osman et al. (27) & 26 & 12 & 7 & 4 & $2.17(1.11,4.23)$ \\
\hline All & 12350 & 9821 & 6775 & 4886 & $1.21(1.05,1.38)$ \\
\hline
\end{tabular}

n.sp.: not specified. 
A All-studies

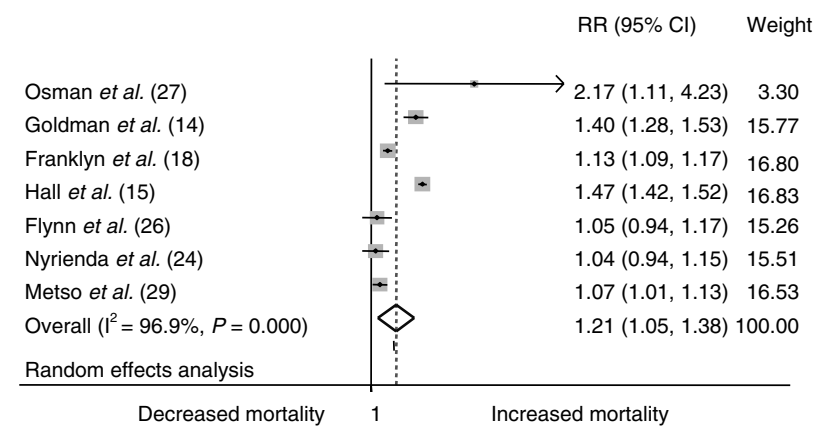

C Comorbidity (cancer and cardiovascular disease)

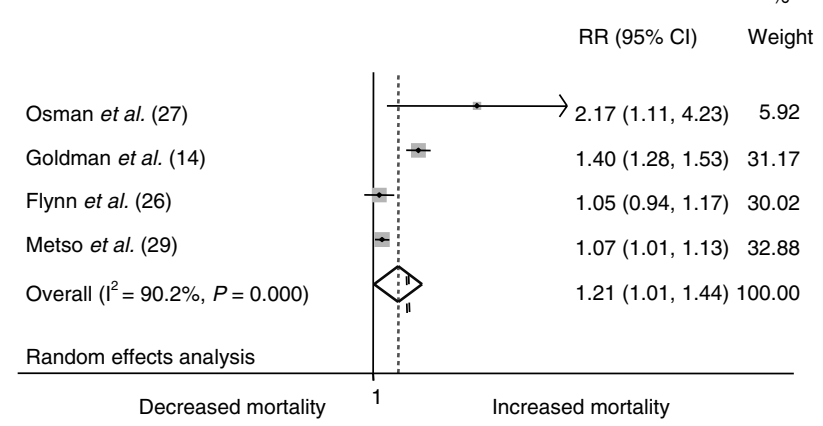

B Treatment modality: radioiodine

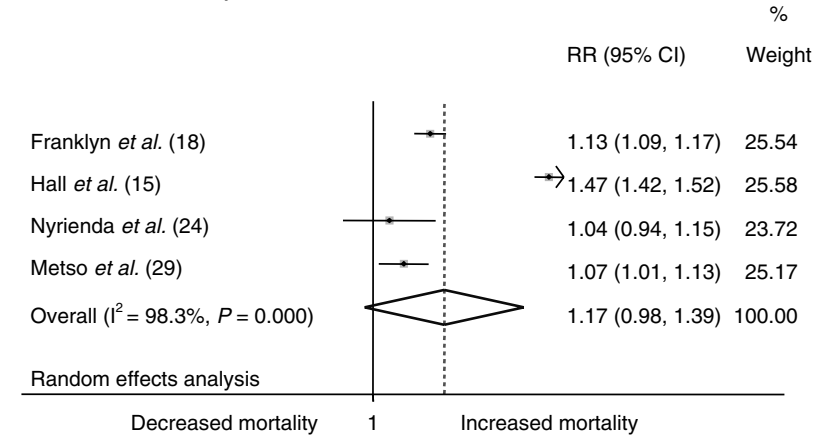

D Cardiovascular mortality

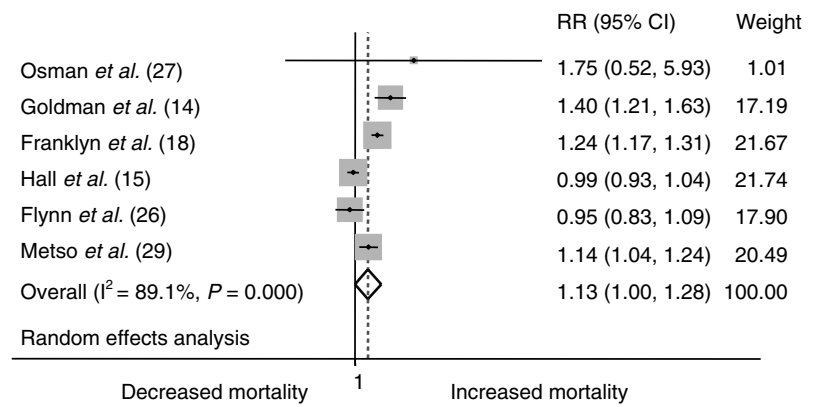

Figure 2 Forrest plot showing the relative risk (RR) of mortality in patients diagnosed with hyperthyroidism. All studies (panel A), stratified for treatment modality (panel B), and co-morbidity (panel C). Panel D shows mortality due to cardiovascular disease.

associated with an increased mortality due to cancer, fractures, respiratory distress, infectious diseases, cardiovascular diseases, and endocrine diseases $(14,18)$. With respect to causality, the association between clinically overt hyperthyroidism and mortality is strong, consistent, biologically plausible, and probably dose dependent. In combination, these features indicate a causal relationship between clinically overt hyperthyroidism and mortality.

Accepting that the association between overt hyperthyroidism and mortality is likely to be causal, the influence of the clinical phenotype and environmental and/or genetic confounders is virtually unknown. In most cases, thyrotoxicosis is due to either Graves' disease or toxic nodular goiter. Clearly, there are major differences in epidemiology and clinical features of these two phenotypes. Graves' disease is the most common cause of hyperthyroidism in iodine-sufficient areas whereas toxic nodular goiter is the dominant cause in iodine-deficient areas (35). The age distribution is also different, toxic nodular goiter often affects older subjects, while Graves' disease is more common in younger subjects. Thus, it is likely that the consequences of these two phenotypes, with respect to mortality, differ. Unfortunately, an evaluation of a possible difference between Graves' disease and toxic nodular goiter with respect to mortality is not possible since it has only been investigated in two studies $(24,29)$.
However, the interpretation of these is unclear, as the study by Nyrienda et al. (24) failed to show a significant association between mortality and any of the two phenotypes. In contrast, the study by Metso et al. (29) demonstrated a significantly increased RR for mortality in patients with toxic nodular goiter, but not in patients with Graves' disease. It follows that future investigations need to clarify the impact of the cause of hyperthyroidism on the association between clinically overt hyperthyroidism and mortality. Another major drawback is the lack of taking environmental and genetic confounders into consideration in the majority of studies. It is important to realize that hyperthyroidism (36), cardiovascular disease (37), lifespan (38), and smoking habits (39) separately demonstrate a familial aggregation. In other words, the association between hyperthyroidism and mortality could very well be due to shared genetic or environmental factors affecting, independently, the development of hyperthyroidism and mortality. Future research should take these factors into account (40).

Importantly, the increased mortality, found in this meta-analysis, is based on an RR estimate. However, this statistical parameter does not specify the time-span between diagnosis and death, number of days or years lost, or whether patients diagnosed with hyperthyroidism tend to die at a younger age. Clearly, a better evaluation of the time-span between diagnosis of 
hyperthyroidism and death will lead to a better understanding of the mechanisms involved. The study by Hall et al. (15) found a 2.65 times higher risk of mortality in the first year after radioiodine treatment, calculated by the standard mortality rate. The value was reduced to 1.49 in the following 8 years. But these data do not allow speculations as to time-span between diagnosis and death, due to the lack of information regarding the delay between diagnosis and treatment.

Finally, since our meta-analysis is based on seven large studies that are inhomogeneous both clinically and methodologically, heterogeneity is to be expected. In line with this, the squared-I values in our analyses range from 89 to $98 \%$, reflecting a high degree of heterogeneity (41). The main reason for this is, however, not related to clinical or methodological differences but to the use of very large cohorts. In the studies included in our meta-analysis $(14,15,18,24$, $26,27,29)$, the risk estimates for death is between 1.04 and 2.17, and due to the large number of patients in each study, the CI of these risk estimates is quite narrow. Thus, although the risk estimates point in the same direction (i.e. increased mortality), there is a statistically significant difference with respect to the size of the risk estimates between studies. It follows that a low squared-I value strengthens the result of a metaanalysis, but still high values do not necessarily arise from pronounced clinically and methodologically heterogeneity.

In conclusion, patients diagnosed with hyperthyroidism have an increased mortality of about $20 \%$ in this meta-analysis. Although the precise mechanism(s) by which hyperthyroidism leads to an increased mortality remains to be defined, the association between clinically overt hyperthyroidism and mortality is strong, relatively consistent, biologically plausible, and probably dose dependent, suggesting that a causal relationship is likely to occur. However, the influence of the type of hyperthyroidism as well as environmental and/or genetic confounders remains to be clarified.

\section{Declaration of interest}

The authors declare that there is no conflict of interest that could be perceived as prejudicing the impartiality of the review reported.

\section{Funding}

F Brandt is enrolled as a Ph.D. student financed by The University of Southern Denmark in Odense. He has also received funding from the Danish Thyroid Patient Organisation. L Hegedüs is the recipient of an unrestricted research grant from the Novo Nordisk Foundation.

\section{References}

1 Wang C \& Crapo LM. The epidemiology of thyroid disease and implications for screening. Endocrinology and Metabolism Clinics of North America 199726 189-218. (doi:10.1016/S08898529(05)70240-1)
2 Bulow PI, Laurberg P, Knudsen N, Jorgensen T, Perrild H, Ovesen L \& Rasmussen LB. Increase in incidence of hyperthyroidism predominantly occurs in young people after iodine fortification of salt in Denmark. Journal of Clinical Endocrinology and Metabolism 200691 3830-3834. (doi:10.1210/jc.2006-0652)

3 Klein I \& Danzi S. Thyroid disease and the heart. Circulation 2007116 1725-1735. (doi:10.1161/CIRCULATIONAHA.106. 678326)

4 Sheu JJ, Kang JH \& Lin HC. Hyperthyroidism and risk of ischemic stroke in young adults: a 5-year follow-up study. Stroke $2010 \mathbf{4 1}$ 961-966. (doi:10.1161/STROKEAHA.109.577742)

5 Lin HC, Yang LY \& Kang JH. Increased risk of pulmonary embolism among patients with hyperthyroidism: a five-year follow-up study. Journal of Thrombosis and Haemostasis $2010 \mathbf{8}$ 2176-2181. (doi:10.1111/j.1538-7836.2010.03993.x)

6 Erem C, Ucuncu O, Yilmaz M, Kocak M, Nuhoglu I \& Ersoz HO. Increased thrombin-activatable fibrinolysis inhibitor and decreased tissue factor pathway inhibitor in patients with hyperthyroidism. Endocrine 200936 473-478. (doi:10.1007/ s12020-009-9271-2)

7 Volzke H, Schwahn C, Wallaschofski H \& Dorr M. Review: the association of thyroid dysfunction with all-cause and circulatory mortality: is there a causal relationship? Journal of Clinical Endocrinology and Metabolism 200792 2421-2429. (doi:10. 1210/jc.2007-0179)

8 Juni P, Witschi A, Bloch R \& Egger M. The hazards of scoring the quality of clinical trials for meta-analysis. Journal of the American Medical Association 1999282 1054-1060. (doi:10.1001/jama. 282.11.1054)

9 DerSimonian R \& Laird N. Meta-analysis in clinical trials. Controlled Clinical Trials 19867 177-188. (doi:10.1016/01972456(86)90046-2)

10 Higgins J, Thompson S, Deeks J \& Altman D. Statistical heterogeneity in systematic reviews of clinical trials: a critical appraisal of guidelines and practice. Journal of Health Services Research and Policy 20027 51-61. (doi:10.1258/1355819021927674)

11 Davey SG, Egger M \& Phillips AN. Meta-analysis. Beyond the grand mean? BMJ $19973151610-1614$.

12 Singer RB. Long-term comparativ mortality in hyperthyroid patients treated with radio-iodine, a cohort study in England. Journal of Insurance Medicine 200133 133-137.

13 Singer RB. Mortality in a complete 4-year follow up of 85-year-old residents of Leiden. Classified by serum level of thyrotropin and thyroxine. Journal of Insurance Medicine 200638 14-19.

14 Goldman MB, Monson RR \& Maloof F. Cancer mortality in women with thyroid disease. Cancer Research $1999502283-2289$.

15 Hall P, Lundell G \& Holm LE. Mortality in patients treated for hyperthyroidism with iodine-131. Acta Endocrinologica 1993128 230-234.

16 Hall P, Berg G, Bjelkengren G, Boice JD Jr, Ericsson UB, Hallquist A, Lidberg M, Lundell G, Tennvall J \& Wiklund K. Cancer mortality after iodine-131 therapy for hyperthyroidism. International Journal of Cancer $1992 \quad \mathbf{5 0}$ 886-890. (doi:10.1002/ijc. 2910500611)

17 Ron E, Doody MM, Becker DV, Brill AB, Curtis RE, Goldman MB, Harris BS III, Hoffman DA, McConahey WM, Maxon HR, PrestonMartin S, Warshauer ME, Wong FL \& Boice JD Jr. Cancer mortality following treatment for adult hyperthyroidism. Cooperative thyrotoxicosis therapy follow-up study group. Journal of the American Medical Association 1998280 347-355. (doi:10.1001/ jama.280.4.347)

18 Franklyn JA, Maisonneuve P, Sheppard MC, Betteridge J \& Boyle P. Mortality after the treatment of hyperthyroidism with radioactive iodine. New England Journal of Medicine 1998338 712-718. (doi:10.1056/NEJM199803123381103)

19 Franklyn JA, Maisonneuve P, Sheppard M, Betteridge J \& Boyle P. Cancer incidence and mortality after radioiodine treatment for hyperthyroidism: a population-based cohort study. Lancet 1999 353 2111-2115. (doi:10.1016/SO140-6736(98)12295-X) 
20 Franklyn JA, Sheppard MC \& Maisonneuve P. Thyroid function and mortality in patients treated for hyperthyroidism. Journal of the American Medical Association 2005294 71-80. (doi:10.1001/ jama.294.1.71)

21 Parle JV, Maisonneuve P, Sheppard MC, Boyle P \& Franklyn JA. Prediction of all-cause and cardiovascular mortality in elderly people from one low serum thyrotropin result: a 10-year cohort study. Lancet $2001 \quad 358 \quad 861-865 . \quad$ (doi:10.1016/S01406736(01)06067-6)

22 Osman F, Gammage MD \& Franklyn JA. Hyperthyroidism and cardiovascular morbidity and mortality. Thyroid 200212 483-487. (doi:10.1089/105072502760143854)

23 Gussekloo J, van EE, de Craen AJ, Meinders AE, Frolich M \& Westendorp RG. Thyroid status, disability and cognitive function, and survival in old age. Journal of the American Medical Association 2004292 2591-2599. (doi:10.1001/jama.292.21.2591)

24 Nyrienda MJ, Clark DN, Finlaysa AR, Read J, Elders A, Brain M, Fox KA \& Toft AD. Thyroid disease and increased cardiovascular risk. Thyroid 200515 718-724. (doi:10.1089/thy.2005.15.718)

25 Cappola AR, Fried LP, Arnold AM, Danese MD, Kuller LH, Burke GL, Tracy RP \& Ladenson PW. Thyroid status, cardiovascular risk, and mortality in older adults. Journal of the American Medical Association 2006295 1033-1041. (doi:10.1001/jama. 295.9.1033)

26 Flynn RW, Macdonald TM, Jung RT, Morris AD \& Leese GP. Mortality and vascular outcomes in patients treated for thyroid dysfunction. Journal of Clinical Endocrinology and Metabolism 2006 91 2159-2164. (doi:10.1210/jc.2005-1833)

27 Osman F, Franklyn JA, Holder RL, Sheppard MC \& Gammage MD. Cardiovascular manifestations of hyperthyroidism before and after antithyroid therapy: a matched case-control study. Journal of the American College of Cardiology 200749 71-81. (doi:10.1016/j. jacc.2006.08.042)

28 Bauer DC, Rodondi N, Stone KL \& Hillier TA. Thyroid hormone use, hyperthyroidism and mortality in older women. American Journal of Medicine 2007120 343-349. (doi:10.1016/j.amjmed. 2006.04.034)

29 Metso S, Jaatinen P, Huhtala H, Auvinen A, Oksala H \& Salmi J. Increased cardiovascular and cancer mortality after radioiodine treatment for hyperthyroidism. Journal of Clinical Endocrinology and Metabolism 200792 2190-2196. (doi:10.1210/jc.2006-2321)

30 Hoffman DA, McConahey WM, Diamond EL \& Kurland LT. Mortality in women treated for hyperthyroidism. American Journal of Epidemiology 1982115 243-254.
31 Hill AB. The environmental and disease: association or causation? Proceedings of the Royal Society of Medicine $1965 \mathbf{5 8} 295-300$.

32 Dorr M, Wolff B, Robinson DM, John U, Ludemann J, Meng W, Felix SB \& Volzke H. The association of thyroid function with cardiac mass and left ventricular hypertrophy. Journal of Clinical Endocrinology and Metabolism 200590 673-677. (doi:10.1210/ jc.2004-1554)

33 Roth RN \& McAuliffe MJ. Hyperthyroidism and thyroid storm. Emergency Medicine Clinics of North America $19897873-883$.

34 Ochs N, Auer R, Bauer DC, Nanchen D, Gussekloo J, Cornuz J \& Rodondi N. Meta-analysis: subclinical thyroid dysfunction and the risk for coronary heart disease and mortality. Annals of Internal Medicine $2008 \mathbf{1 4 8} 832-845$.

35 Laurberg P, Pedersen KM, Hreidarsson A, Sigfusson N, Iversen E \& Knudsen PR. Iodine intake and the pattern of thyroid disorders: a comparative epidemiological study of thyroid abnormalities in the elderly in Iceland and in Jutland, Denmark. Journal of Clinical Endocrinology and Metabolism 199883 765-769. (doi:10.1210/ jc.83.3.765)

36 Brix TH, Kyvik KO, Christensen K \& Hegedus L. Evidence for a major role of heredity in Graves' disease: a population-based study of two Danish twin cohorts. Journal of Clinical Endocrinology and Metabolism 200186 930-934. (doi:10.1210/jc.86.2.930)

37 Acton RT, Go RC \& Roseman JM. Genetics and cardiovascular disease. Ethnicity and Disease 200414 2-16.

38 Herskind AM, McGue M, Holm NV, Sorensen TI, Harvald B \& Vaupel JW. The heritability of human longevity: a populationbased study of 2872 Danish twin pairs born 1870-1900. Human Genetics 200697 319-323. (doi:10.1007/BF02185763)

39 Madden PA, Pedersen NL, Kaprio J, Koskenvuo MJ \& Martin NG. The epidemiology and genetics of smoking initiation and persistence: crosscultural comparisons of twin study results. Twin Research 20047 82-97. (doi:10.1375/13690520460741471)

40 Martin N, Boomsma D \& Machin G. A twin-pronged attack on complex traits. Nature Genetics 199717 387-392. (doi:10.1038/ ng1297-387)

41 Higgins JP, Thompson SG, Deeks JJ \& Altman DG. Measuring inconsistency in meta-analyses. BMJ $2003 \mathbf{3 2 7} 557-560$. (doi:10.1136/bmj.327.7414.557)

Received 17 June 2011

Accepted 1 July 2011 